\title{
Survival analysis approach to account for non-exponential decay rate effects in lifetime experiments
}

\author{
K. J. Coakley ${ }^{\mathrm{a}, *}$, M. S. Dewey ${ }^{\mathrm{b}}$, M. G. Huber ${ }^{\mathrm{b}}$, P. R. Huffman ${ }^{\mathrm{c}, \mathrm{d}}$, C. R. \\ Huffer $^{\mathrm{c}, \mathrm{d}}$, D. E. Marley ${ }^{\mathrm{b}, \mathrm{c}}$, H. P. Mumm ${ }^{\mathrm{b}}$, C. M. O’Shaughnessy ${ }^{\mathrm{e}, \mathrm{d}}$, K. W. \\ Schelhammer ${ }^{\mathrm{c}, \mathrm{d}}$, A. K. Thompson ${ }^{\mathrm{b}}$, A. T. Yue ${ }^{\mathrm{b}}$ \\ ${ }^{a}$ National Institute of Standards and Technology, 325 Broadway, Boulder, CO 80305 \\ ${ }^{b}$ National Institute of Standards and Technology, 100 Bureau Drive, Stop 8461, \\ Gaithersburg, MD 20899 \\ ${ }^{c}$ North Carolina State University, 2401 Stinson Drive, Box 8202, Raleigh, NC 27695 \\ ${ }^{d}$ Triangle Universities Nuclear Laboratory, 116 Science Drive, Box 90308, Durham, NC \\ 27708 \\ ${ }^{e}$ University of North Carolina at Chapel Hill, 120 E. Cameron Ave., CB \#3255, Chapel \\ Hill, NC 27599
}

\begin{abstract}
In experiments that measure the lifetime of trapped particles, in addition to loss mechanisms with exponential survival probability functions, particles can be lost by mechanisms with non-exponential survival probability functions. Failure to account for such loss mechanisms produces systematic measurement error and associated systematic uncertainties in these measurements. In this work, we develop a general competing risks survival analysis method to account for the joint effect of loss mechanisms with either exponential or non-exponential survival probability functions, and a method to quantify the size of systematic effects and associated uncertainties for lifetime estimates. As a case study, we apply our survival analysis formalism and method to the Ultra Cold Neutron lifetime experiment at NIST. In this experiment, neutrons can escape a magnetic trap before they decay due to a wall loss mechanism with an associated non-exponential survival probability function.
\end{abstract}

Keywords: Chaotic scattering

\footnotetext{
*Corresponding author

Email address: kevincoakley@nist.gov (K. J. Coakley)
}

Preprint submitted to NIMA

December 13, 2015 
Competing risks, Magnetic trapping, Marginally trapped neutrons, Monte Carlo simulation, Neutron lifetime, Stochastic modeling, Survival analysis, Symplectic integration

PACS: code 81.07.Gf, 07.05.Kf, 07.79.-v, 07.79.Lh

\section{Introduction}

According to the Particle Data Group, the current best estimate of the mean lifetime of the neutron $\tau_{n}$ is $(880.1 \pm 1.1) \mathrm{s}$ [1]. The difference between neutron lifetime estimates determined by beam and bottle experiments is $(8.4 \pm 2.2)$ s. This difference is likely due to unresolved systematic errors [2]. Hence, reduction of systematic uncertainties is key to reducing the overall uncertainty of the mean lifetime of the neutron [3]. In trapping experiments, marginally trapped neutrons can escape a trapping volume before they $\beta$-decay [4-9]. In general, we expect the survival probability functions associated with these loss mechanisms to be non-exponential. Accounting for systematic effects due to these loss mechanisms is the primary motivation for the methodological work presented here.

As far as we know, there are no analytical methods to model experimental data for lifetime experiments where particles are affected by multiple loss mechanisms with exponential and non-exponential survival probability functions. We develop an analytical model that jointly accounts for both types of loss mechanisms with a competing risks survival analysis model $[10,11,12,13]$. In this approach, we assume random loss times due to different mechanisms are mutually independent. Our method predicts the particle loss rates for each of many loss mechanisms and enables one to construct prediction models for experimental data given knowledge of which mechanisms produce detectable signals. Based on our methods, one can construct algorithms to directly estimate the mean lifetime (due to the joint effect of loss mechanisms with exponential survival probability functions) when the expected number of trapped particles is a non-exponential function of time. Further, our theoretical model accounts for convolution effects due to random creation (or injection) times of particles into the trap. As a caveat, our method depends on theoretical knowledge of the production (or injection) rate of particles during a filling stage and knowledge of a non-exponential survival probability associated with the loss mechanisms responsible for nonexponential detection rates. 
To illustrate our method, we apply it to a subset of data from a magnetic trapping experiment at NIST $[5,6]$. Several of the specific systematic effects discussed are relevant to other neutron trapping experiments. In the NIST experiment, an Ultra Cold Neutron (UCN) is produced during a trap-filling stage when a $12 \mathrm{~K}$ neutron is scattered to near rest in liquid helium by single phonon emission [14]. After filling the trap and blocking the cold beam that produces UCNs, light produced by neutron decay and background events is detected by a pair of phototubes. A marginally trapped (or above threshold) neutron is an UCN with sufficient energy to escape the trap by interacting with materials at the boundary of the trap $[15,16,17]$. In contrast, a UCN with insufficient energy to escape the trap is a below threshold UCN. Since the probability of losing a neutron when it interacts with materials at a wall depends, in part, on neutron energy, the survival probability of a marginally trapped UCN is non-exponential. Hence, when wall losses are non-negligible, on average, the observed $\beta$-decay rate of neutrons is also non-exponential. Thus, if one were to fit an exponential model to such decay event data, one would expect the associated estimate of the neutron lifetime to be biased. A key input to the competing risks survival analysis model is the survival probability function associated with the wall loss mechanism. Based on a physical model for how neutrons interact with materials at the walls of the trap, we determine this survival probability function with a Monte Carlo method as described in [18]. We then construct a prediction model for the observed NIST data. If $\beta$-decay and wall losses were the only loss mechanisms, and we had perfect knowledge of the survival probability of marginally trapped UCNs as well as possible backgrounds, a fit of this model to data would yield a nearly unbiased estimate of the true neutron lifetime given a sufficient amount of data. In practice, there are other loss mechanisms including upscattering and neutron absorption by ${ }^{3} \mathrm{He}$ that affect the mean lifetime of neutrons in the trap. We stress that systematic error (and associated systematic uncertainty) associated with marginally trapped UCNs can be reduced by field ramping strategies to very low levels. Hence, the systematic error we report for the case presented here (a static potential) does not apply to cases where marginally trapped UCNs are purged by field ramping methods. The static runs enable us to test the validity of our models and simulation methods.

In most runs of the NIST experiment, we vary the trapping potential to purge marginally trapped UCNs. In a simulation experiment, we demonstrate that field ramping can in principle reduce systematic measurement 
error and associated uncertainty due to marginally trapped UCNs to very low levels at the expense of purging below threshold UCNs. For a particular example, we demonstrate that the above ramping strategy is more efficient than one where neutrons are trapped when the field is fully ramped and then increased to its maximum value. By more efficient, we mean that the expected number of below threshold UCNs after ramping is higher for our ramping strategy as compared to the alternative strategy given that both methods purge marginally trapped UCNs with nearly the same efficiency.

In this paper, we first develop a general competing risks survival analysis model that applies to the NIST experiment as well as related experiments. In an experimental application section, we apply our survival analysis methods to the NIST experimental data. We first discuss physical and computational methods for simulating UCN trajectories and loss probabilities of UCNs due to wall interactions. Based on our survival analysis prediction model, we estimate the mean lifetime of trapped neutrons. We also quantify uncertainties associated with Monte Carlo sampling variability and imperfect knowledge of physical models for neutron interactions with materials at the walls of trap and beam divergence effects. Finally, in a Monte Carlo simulation experiment, we demonstrate that field ramping can reduce systematic error due to wall losses of UCN to a very low level at the expense of reducing the expected number of trapped UCNs after ramping.

\section{Survival Analysis Model}

\subsection{Mathematical preliminaries}

In survival analysis, for any loss mechanism, the loss time $T$ of an object (in our case a neutron) created at time $t=0$ is a random variable with survival probability $S(t)$ where

$$
S(t)=\operatorname{Pr}(T>t) .
$$

For a continuous $S(t)$, one can define the hazard function $\lambda(t)$ which is the instantaneous loss rate at time $t$ given that the object of interest survives until time $t$ as follows.

$$
\lambda(t)=\lim _{\Delta t \rightarrow 0} \frac{\operatorname{Pr}(t \leq T \leq t+\Delta t \mid T \geq t)}{\Delta t},
$$

where $\operatorname{Pr}(t \leq T \leq t+\Delta t \mid T \geq t)$ is the conditional probability that the loss time $T$ falls in the interval $[t, t+\Delta t]$ given that $T$ is no less than $t$. Based on 
the well known conditional probability equality $\operatorname{Pr}(A \mid B)=\operatorname{Pr}(A \cap B) / \operatorname{Pr}(B)$, one gets the following well known expression for the hazard function

$$
\lambda(t)=\frac{1}{S(t)} \lim _{\Delta t \rightarrow 0} \frac{S(t)-S(t+\Delta t)}{\Delta t}=-\frac{\mathrm{d} \log S(t)}{\mathrm{d} t} .
$$

For instance, for the neutron $\beta$-decay loss mechanism, the associated hazard function is $\lambda_{\beta}=\frac{1}{\tau_{n}}$ where $\tau_{n}$ is the neutron lifetime. In general, for any loss mechanism,

$$
S(t)=\exp (-\Lambda(t))
$$

where the cumulative hazard function $\Lambda(t)$ is

$$
\Lambda(t)=\int_{t=0}^{t} \lambda(t) \mathrm{d} t .
$$

Thus, the survival probability function associated with $\beta$-decay is $S_{\beta}(t)=$ $\exp \left(-\lambda_{\beta} \tau_{n}\right)=\exp \left(-\frac{t}{\tau_{n}}\right)$.

In a competing risks model, the multivariate survival probability function for the case of $K$ loss mechanisms is $S\left(t_{1}, t_{2}, \cdots, t_{K}\right)$ where

$$
S\left(t_{1}, t_{2}, \cdots, t_{K}\right)=\operatorname{Pr}\left(T_{1} \geq t_{1}, T_{2} \geq t_{2}, \cdots T_{K} \geq t_{K}\right) .
$$

The term $T_{i}$ is a random variable representing the loss time associated with the $i$ th loss mechanism. In general, for $i \neq j$, the random variables $T_{i}$ and $T_{j}$ need not be independent. The actual loss time of the object of interest is $T=\min \left(T_{1}, T_{2}, \cdots T_{K}\right)$. The cause-specific hazard function for the $j$ th loss mechanism at time $t, \lambda_{j}$, is

$$
\lambda_{j}(t)=-\left.\frac{\partial \log S}{\partial t_{j}}\right|_{t_{1}=t_{2}=\cdots t_{K}=t}
$$

Further,the overall hazard function and survival probability function at time $t$ are $\sum_{j=1}^{K} \lambda_{j}(t)$ and $S\left(t_{1}=t, t_{2}=t, \cdots, t_{K}=t\right)=\exp \left(-\sum_{j=1}^{K} \Lambda_{j}(t)\right)$ respectively. In this work, we assume that loss times due to different mechanisms are mutually independent. Given this independence assumption, we can write $S\left(t_{1}, t_{2}, \cdots, t_{K}\right)=\prod_{j=1}^{K} S_{j}\left(t_{j}\right)$ where $S_{j}(t)$ is the survival probability function associated with the $j$ th loss mechanism. Hence, the cause-specific loss mechanism for the $j$ th loss mechanism is

$$
\lambda_{j}(t)=-\frac{\mathrm{d} \log S_{j}(t)}{\mathrm{d} t} .
$$




\subsection{Conditional survival probability}

In experiments like the NIST magnetic trapping experiment detailed later in this work, particles of interest are created (or perhaps injected) during a filling stage of fixed duration. Suppose that the filling stage occurs during the time interval $\left[0, t_{L}\right] \mathrm{s}$. Here, we model the cumulative number of created particles at time $t \leq t_{L}$ as a realization of a inhomogeneous Poisson process with time-varying rate parameter $g(t)$. For the first loss mechanism, the conditional survival probability for particles that are randomly created during the filling stage and that survive the filling stage is

$$
\begin{gathered}
S_{1}\left(t \mid T \geq t_{L}\right)=\left[\int_{s=0}^{s=t_{L}} g(s) S\left(t-s, t_{L}-s, \cdots, t_{L}-s\right) d s\right] / \\
{\left[\int_{s=0}^{s=t_{L}} g(s) S\left(t_{L}-s, t_{L}-s, \cdots, t_{L}-s\right) d s\right] .}
\end{gathered}
$$

Similar formulae apply for the other loss mechanisms. Further, the conditional cause-specific loss mechanism for the $j$ th loss mechanism for these particles is

$$
\lambda_{j}\left(t \mid T \geq t_{L}\right)=-\frac{\mathrm{d} \log S_{j}\left(t \mid T \geq t_{L}\right)}{\mathrm{d} t} .
$$

The expected number of surviving particles at time $t_{L}$ is

$$
<N\left(t_{L}\right)>=\int_{s=0}^{s=t_{L}} g(s) S\left(t_{L}-s, t_{L}-s, \cdots, t_{L}-s\right) d s .
$$

If there are $K$ loss mechanisms, the expected number of surviving particles at time $t \geq t_{L}$ is

$$
<N(t)>=<N\left(t_{L}\right)>\prod_{k=1}^{K} S_{k}\left(t \mid T \geq t_{L}\right) .
$$

Finally, the theoretical rate at which particles are lost by the $j$ th loss mechanisms at $t \geq t_{L}$ is

$$
r_{j}\left(t \mid T \geq t_{L}\right)=<N(t)>\lambda_{j}\left(t \mid T \geq t_{L}\right) .
$$

Some loss mechanisms will generate detectable signals. Other loss mechanisms may or may not. Based on predicted rates corresponding to loss 
mechanisms that produce detectable signals, one can construct a prediction model for observed data and directly estimate the mean lifetime (due to loss mechanisms with exponential survival probability functions) of a trapped particle. In the next section, we develop such procedures for analysis of observed data acquired in a magnetic trapping experiment at NIST. We estimate the survival probability function associated with wall losses based on a physical model for neutron interactions with materials at the boundary of the trap by a Monte Carlo method. In our analysis, neutrons with energies below a threshold are not affected by wall losses. Hence, to maximize computational resources, we split simulated neutrons into above threshold and below threshold sub-samples and estimate the survival probability for the wall loss mechanism only for the above threshold subsample.

\section{Experimental Application}

\subsection{Trapping potential model}

In the NIST experiment, an UCN with sufficiently low energy is trapped in the potential field produced by the interaction of the magnetic moment of a neutron and a spatially varying magnetic field (Figure 1) and gravity. For tracking UCNs in the trapping volume, assuming adiabatic spin transport, the potential is

$$
V(\mathbf{x})=\mu|\mathbf{B}(\mathbf{x})|+m_{n} g y,
$$

where $\mathbf{B}$ is the magnetic field, $m_{n}$ and $\mu$ are the mass and magnetic moment of the neutron respectively, $\mathbf{x}=(x, y, z)$ is spatial location of the neutron in the Cartesian coordinates shown in Figure 1, and $g$ is the acceleration due to gravity.

We define a nominal trapping volume corresponding to $-z_{o} \leq z \leq z_{o}$ where $z$ is the axial coordinate and $z_{o}=37.5 \mathrm{~cm}$ (see Figure 1). An UCN with total energy (kinetic plus potential) greater than the minimum of the potential $V_{\min }$ on the boundary of the nominal trapping volume is defined to be a marginally trapped (or above threshold) UCN. An UCN with energy less than or equal to $V_{\min }$ is a below threshold UCN. We stress that there is not a physical boundary at $z=-37.5 \mathrm{~cm}$. However, as shown in Figure 1 , as $z$ is reduced from $z \approx-37.5 \mathrm{~cm}$ to $-60 \mathrm{~cm}$, the trapping potential decreases dramatically. For modeling purposes and to speed up Monte Carlo simulations, it is important to define a nominal trapping volume so that all UCN with initial energy less than the minimum value trapping potential on 
the boundary of the nominal trapping volume never interact with materials at $z=-60 \mathrm{~cm}$ and $z=37.5 \mathrm{~cm}$, nor the cylindrical boundary $r=5.6 \mathrm{~cm}$. If we choose the lower axial boundary to be much less than $-37.5 \mathrm{~cm}$, this condition is violated. Different choices of the minimum axial location of the nominal trapping volume affect results. In Section 3.7, we quantify this effect. For a typical static trap configuration of the NIST experiment, $V_{\min }=139$ $\mathrm{neV}$. We assume that probability distribution function of the initial speed $|v|$ of an UCN produced in the trap has a quadratic form [18,19] $f(|v|) \propto|v|^{2}$ for low velocities of interest.

We determine a neutron trajectory based on its initial position and momentum, by solving the classical equations of motion

$$
\dot{\mathbf{p}}=F(\mathbf{x})=-\nabla \mathbf{V}(\mathbf{x}),
$$

and

$$
\dot{\mathbf{x}}=\frac{\mathbf{p}}{m_{n}},
$$

with an optimal fourth order symplectic integration scheme [18,19,20,21]. We predict $|\mathbf{B}|$ at arbitrary points in the trapping volume with a threedimensional tensor-product spline interpolant [22] where the order of the spline is four in each direction. We determine the tensor-product B-spline coefficients from values of $|\mathbf{B}|$ computed on a grid by a code that solves the Biot-Savart law numerically based on the geometry of the solenoid and current bars that produce the magnetic field. With this tensor-product method, we evaluate the potential and its gradient at arbitrary locations within the trap.

In some experiments, we ramp the quadrupole field $\mathbf{B}_{q}$ while keeping the solenoid field $\mathbf{B}_{s}$ constant. Given that the filling stage ends at time $t_{L}$, the ramping factor for the quadrupole field is $R\left(t-t_{L}\right)$, the magnitude of the B-field varies as

$$
\left|\mathbf{B}\left(t-t_{L}\right)\right|=\left|\mathbf{B}_{s}+R\left(t-t_{L}\right) \mathbf{B}_{q, \max }\right|,
$$

where $\mathbf{B}_{q, \max }$ is the maximum value of the quadrupole field. For ramping cases, we develop a tensor-spline method to model the gradient of $\left|\mathbf{B}\left(t-t_{L}\right)\right|$ based on tensor-spline models for each component of $\mathbf{B}_{s}$ and $\mathbf{B}_{q, \max }$.

\subsection{Wall loss model}

When an above threshold UCN collides with the cylindrical wall or endcap boundaries, we assign it a loss probability $p_{\text {loss }}$ according to a model based 
on assumed material properties $[15,16,17]$. After $k$ collisions, the empirical survival probability of the $\mathrm{UCN}$ is

$$
p_{\text {surv }}(k)=\prod_{i=1}^{k}\left[1-p_{\text {loss }}(i)\right] .
$$

We track each above threshold UCN until its empirical survival probability drops below $10^{-9}$. Due to chaotic scattering effects, the symplectic integration prediction for a trajectory of an UCN does not converge in general as the time step parameter in the integration code is reduced [19]. Here, we assume that the mean survival probability at any time $t$ for an ensemble of UCNs does not depend on the time step parameter even though the predicted survival probability of a particular UCN may depend on the time step parameter. Although we are unaware of any proof that this assumption is true, it seems reasonable. For more discussion of this point, see [19].

We model the interaction of the neutron with the materials on the trap boundaries with a one-dimensional optical model based on Schrodinger's equation. In this approach, we assume that each neutron energy is sufficiently low so that coherent effects are significant and interactions are well predicted by an optical model where the neutron potential is $V-i W$. For the cylindrical walls, we model the the neutron potential as due to layers of different homogenous materials following [15]. The materials at the endcaps at $z=-60 \mathrm{~cm}$ and $z=37.5 \mathrm{~cm}$ are Teflon FEP ${ }^{1}$ and acrylic respectively. Given that a UCN with velocity $\mathbf{v}$ crosses the trap boundary at location $\mathbf{x}$ and that the surface normal for the trap boundary at $\mathbf{x}$ is $\hat{\mathbf{n}}$, we define $E_{\perp}=\frac{1}{2} m_{n}|\mathbf{v} \cdot \hat{\mathbf{n}}|^{2}$. For the endcaps, we model the probability of diffuse reflection off the wall based on Eq. 2.71 of [14] as

$$
p_{\text {scat }}=\frac{E_{\perp}-\sqrt{E_{\perp}\left(2 \alpha-2\left(V_{*}-E_{\perp}\right)\right)}+\alpha}{E_{\perp}+\sqrt{E_{\perp}\left(2 \alpha-2\left(V_{*}-E_{\perp}\right)\right)}+\alpha},
$$

where

$$
\alpha=\sqrt{\left(V_{*}-E_{\perp}\right)^{2}+W^{2}}
$$

\footnotetext{
${ }^{1}$ Certain materials are identified in this paper to foster understanding. Such identification does not imply recommendation or endorsement by the National Institute of Standards and Technology, nor does it imply that the materials identified are necessarily the best available for the purpose.
} 
and $V_{*}=V-V_{H e}$ where $V_{H e}=15.98 \mathrm{neV}$. For the Teflon material, $V=27.8$ $\mathrm{neV}$ and $W=1.39 \mathrm{e}-04 \mathrm{neV}$. For the acrylic material, $V=121.04 \mathrm{neV}$ and $W=4.74 \mathrm{e}-05 \mathrm{neV}$.

The material that coats the cylindrical walls of the trap is modeled as multilayer of tetraphenyl butadiene (TPB) $\left(\mathrm{C}_{28} \mathrm{H}_{22}\right)$, Gore-Tex $\left(\mathrm{C}_{2} \mathrm{~F}_{4}\right)$, graphite $(\mathrm{C})$, and boron nitride $(\mathrm{BN})$. The TPB used in the experiment is not deuterated and therefore contains a substantial amount of hydrogen, which has a very large incoherent scattering cross section. Since the optical model does not account for incoherent scattering, we add an additional term to account for it. The real and imaginary components of the augmented potential for each multilayer are

$$
V=\frac{2 \pi \hbar^{2}}{m_{n}} \sum_{i} N_{i} a_{i},
$$

and

$$
W=\frac{\hbar v}{2} \sum_{i} N_{i}\left(\sigma_{\text {abs }}^{i}+\sigma_{\text {loss }}^{i}\right),
$$

where for the $i$ th nuclear isotope, $N_{i}$ is nucleus density; $a_{i}$ is the coherent scattering length; $\sigma_{\text {abs }}^{i}$ is the absorption cross section; and $\sigma_{\text {loss }}^{i}$ accounts for incoherent scattering losses. Since approximately half of the neutrons that undergo incoherent scattering will be scattered back into the ${ }^{4} \mathrm{He}$ and the rest will be lost, $\sigma_{\text {loss }}$ is set to half of the estimated total incoherent cross section. After this modification, we solve the appropriate differential equation and determine the loss probability of the neutron as a function of $E_{\perp}$ (Figure 2). Our model does not include the possibility of surface contamination [23] as we do not currently have a way of characterizing surface contamination quantitatively. However, for the most part, such contamination would lead to an additional marginally-trapped neutron loss mechanism. We expect that such an additional loss mechanism would reduce the systematic effect associated with marginally trapped neutrons.

After each wall collision, we scatter the neutron back into the trapping volume. Since the surface of the walls that scatter neutrons is rough, we model the reflection of the neutron with a Lambertian model [24] rather than a specular model that is appropriate for a perfectly smooth surface. In optical applications of the Lambertian model, the intensity of a reflected signal is proportional to $\cos (\theta)$ where $\theta$ is the inclination angle between the surface normal of the emitter and the direction of the reflected radiation. Hence, in our simulation studies, it follows that the cumulative distribution 
function $(\mathrm{CDF})$ for the inclination angle between the surface normal of the wall and the direction of reflected neutron $\theta$ is

$$
F_{L}(\theta)=\frac{1-\cos (2 \theta)}{2}
$$

where $0 \leq \theta \leq \frac{\pi}{2}$. The azimuthal angle $\phi$ is a uniformly distributed random variable between 0 and $2 \pi$. In contrast, for a diffuse reflection model based on a uniform intensity model, the CDF of the direction cosine of the reflected neutron is

$$
F_{U}(\cos (\theta))=\cos (\theta)
$$

where $0 \leq \cos (\theta) \leq 1$. Later, in Section 3.7, we quantify the variation of the estimated lifetime of trapped neutrons for different neutron reflectivity models.

\subsection{Survival analysis of NIST experiment}

In the NIST experiment, we assume that the creation rate $g(t)$ of UCNs during the filling stage is constant. Hence, we model the creation time of any UCN during the filling stage as a uniform random variable between $t=0$ and $t=t_{L}$ where $t_{L}$ is the time spent filling (loading) the trap. Aside from $\beta$-decay and wall losses, neutrons can be lost by absorption processes associated with impurities (primarily ${ }^{3} \mathrm{He}$ capture ) and by upscattering. We expect the hazard function associated with upscattering to vary with temperature. However, for the ideal case where temperature is constant, the hazard function for upscattering is a constant $\lambda_{u}=\frac{1}{\tau_{u}}$. From first principle arguments, the hazard function associated with an energy-independent absorption

process is a constant $\lambda_{a}=\frac{1}{\tau_{a}}$. We define $\lambda_{*}$ to be sum of hazard functions associated with $\beta$-decay, upscattering and an additional energy-independent absorption process. We assume that the hazard function associated with upscattering does not vary with time. Hence,

$$
\lambda_{*}=\frac{1}{\tau_{*}}=\frac{1}{\tau_{n}}+\frac{1}{\tau_{u}}+\frac{1}{\tau_{a}}
$$

is a constant in our model. We stress that $\lambda_{*}$ does not account for the time-varying hazard function (and associated non-exponential survival probability) associated with the wall loss mechanism. Next, we develop a model that enables us to directly estimate $\lambda_{*}$ from experimental data given that we 
have an estimate for the survival probability function $S_{M}(t)$ associated with the wall loss mechanism.

Following arguments in [18], given the production rate of below and above threshold UCNs during the filling stage are $r_{-}$and $r_{+}$, we predict the expected number of below threshold UCNs at the end of filling stage $\left(t_{L}\right)$ as

$$
\left\langle N_{-}\left(t_{L}\right)\right\rangle=r_{-} \int_{s=0}^{t_{L}} \exp \left(\lambda_{*}\left(s-t_{L}\right)\right) d s .
$$

The predicted number of above threshold UCNs at the end of the filling stage is

$$
\left\langle N_{+}\left(t_{L}\right)\right\rangle=r_{+} \int_{s=0}^{t_{L}} S_{M}\left(t_{L}-s\right) \exp \left(\lambda_{*}\left(s-t_{L}\right)\right) d s,
$$

where $S_{M}(t)$ is survival probability function associated with wall losses of above threshold UCNs. As stated earlier, $S_{M}(t)$ has a non-exponential form in general.

Given that an above threshold UCN survives the filling stage, the conditional survival probability associated with wall losses is

$$
\begin{gathered}
S_{M}^{+}(t)=S_{M}\left(t \mid T \geq t_{L}\right)=\left[\int_{s=0}^{t_{L}} S_{M}(t-s) \exp \left(\lambda_{*}\left(s-t_{L}\right)\right) d s\right] / \\
{\left[\int_{s=0}^{t_{L}} S_{M}\left(t_{L}-s\right) \exp \left(\lambda_{*}\left(s-t_{L}\right)\right) d s\right] .}
\end{gathered}
$$

For loss mechanisms with exponential survival probability functions, $S(t \mid T>$ $\left.t_{L}\right)=S\left(t-t_{L}\right)$. Hence, for times $t>t_{L}$, the expected number of UCNs in the trap,

$$
\langle N(t)\rangle=\left\langle N_{-}\left(t_{L}\right)\right\rangle\left[1+\frac{\left\langle N_{+}\left(t_{L}\right)\right\rangle}{\left\langle N_{-}\left(t_{L}\right)\right\rangle} S_{M}^{+}(t)\right] \exp \left(-\lambda_{*}\left(t-t_{L}\right)\right) .
$$

We can rewrite the above as

$$
\langle N(t)\rangle=\left\langle N_{-}\left(t_{L}\right)\right\rangle[1+\Delta(t)] \exp \left(-\lambda_{*}\left(t-t_{L}\right)\right),
$$

where the time-dependent "distortion" term $\Delta$ is

$$
\Delta(t)=\frac{\left\langle N_{+}\left(t_{L}\right)\right\rangle}{\left\langle N_{-}\left(t_{L}\right)\right\rangle} S_{M}^{+}(t)
$$


Based on the above, the expected loss rate of neutrons lost due to $\beta$-decay, absorption, and upscattering are $r_{\beta}(t)=\frac{\langle N(t)\rangle}{\tau_{n}}, r_{a}(t)=\frac{\langle N(t)\rangle}{\tau_{a}}$, and $r_{u}(t)=$ $\frac{\langle N(t)\rangle}{\tau_{u}}$ respectively. Given that upscattering losses are unobservable and absorption events yield events that are indistinguishable from $\beta$-decay events, the overall predicted detection rate is

$$
r_{d e t}(t)=\langle N(t)\rangle\left[\frac{p_{\beta}}{\tau_{n}}+\frac{p_{a}}{\tau_{a}}\right]
$$

where $p_{\beta}$ and $p_{a}$ are detection probabilities for two loss mechanisms.

\subsection{Contamination ratio}

Following [18], we can decompose $\langle N(t)\rangle$ into exponential and non-exponential components as follows.

$$
\langle N(t)\rangle=f_{\exp }(t)+f_{c}(t)
$$

where

$$
f_{\text {exp }}(t)=\left\langle N_{-}\left(t_{L}\right)\right\rangle\left[1+\Delta\left(t_{\text {end }}\right)\right] \exp \left(-\lambda_{*}\left(t-t_{L}\right)\right)
$$

and

$$
f_{c}(t)=\left\langle N_{-}\left(t_{L}\right)\right\rangle\left[\Delta(t)-\Delta\left(t_{\text {end }}\right)\right] \exp \left(-\lambda_{*}\left(t-t_{L}\right)\right) .
$$

The ratio of the non-exponential and exponential terms can be regarded as a contamination ratio $r_{c}$ where

$$
r_{c}(t)=\frac{\Delta(t)-\Delta\left(t_{\text {end }}\right)}{1+\Delta\left(t_{\text {end }}\right)} .
$$

We emphasize that $\Delta(t)$ and $f_{c}(t)$ are nonlinear functions of $\lambda_{*}$. In Section 3.7, we estimate $\lambda_{*}$ directly from experimental data given a Monte Carlo estimate of $S_{M}(t)$ based on a physical model for the wall loss probability and how surviving neutrons are reflected back into the trapping volume. The uncertainty of $\lambda_{*}$ depends, in part, on imperfect knowledge of: the wall loss probability model; how surviving neutrons reflect off the walls; the spatially varying fluence of the thermal beam that produces UCN in the trap; and the usual counting statistics variability in the observed data. 


\subsection{Prediction model}

For experimental data corresponding to a particular subset of runs from the NIST experiment, we estimate $\lambda_{*}$ by fitting a model to experimental data based on Eqns. 19 and 21. The primary (background plus neutron events) data are acquired for a static trapping potential. Background measurements are also acquired in non-trapping runs and subtracted from the primary observations [5]. Our primary goal is to understand systematic measurement errors and associated uncertainties associated with marginally trapped neutrons and other loss mechanisms. Hence, we analyze data corresponding to an experiment where we did not ramp the magnetic field in order to maximize the systematic effects of marginally trapped neutrons.

More specifically, for bins that are $1 \mathrm{~s}$ wide, we predict the number of background-corrected counts in the $k$ th bin as

$$
\hat{n}_{k}=A \lambda_{*} \delta_{t}\left[1+\Delta\left(t_{k}\right)\right] \exp \left(-\lambda_{*}\left(t_{k}-t_{L}\right)\right)+r_{b g} \delta_{t}
$$

where $\delta_{t}=1 \mathrm{~s}$ is the resolution at which we determine survival probabilities by our Monte Carlo method, $t_{k}$ is the midpoint of the $k$ th time bin, and $A, r_{b g}$ and $\lambda_{*}$ are adjustable model parameters determined by our modeling fitting procedure. Since the width of the bins for the observed data is $15 \mathrm{~s}$, we aggregate predictions at the $1 \mathrm{~s}$ scale to get predictions at the $15 \mathrm{~s}$ scale of interest.

Given that we estimate these parameters to be $\hat{A}$ and $\hat{\lambda}$, we predict the expected number of below threshold trapped UCNs at $t=t_{L}$ as

$$
\left\langle\widehat{N_{-}\left(t_{L}\right)}\right\rangle=\frac{\hat{A} \hat{\lambda}_{*}}{p_{\beta}\left(\hat{\lambda}_{*}-\lambda_{a}-\lambda_{u}\right)+p_{a} \lambda_{a}}
$$

where the hazard functions $\lambda_{a}$ and $\lambda_{u}$ and detection probabilities $p_{a}$ and $p_{\beta}$ are determined from other experiments and/or theoretical arguments.

\subsection{Estimation details}

In the NIST experiment, the cold neutron beam that produces the UCNs is collimated. Based on an uncollimated measured beam profile and knowledge of the geometry of the collimator, we estimate a spatially varying neutron fluence image at the entrance to the detector (Figure 3) and an associated probability density function for the intersection of any neutron trajectory and the plane at $z=-60 \mathrm{~cm}$. Based on this probability density 
function, we simulate intersection points with the Von Neuman rejection sampling method $[25,26]$. For each intersection point, we simulate a neutron velocity direction vector $\hat{v}$ that has length 1 . For the case where there is no beam divergence, $\hat{v}$ is parallel to the axial direction of the trap. For the case of non-zero divergence, we simulate $\hat{v}$ such that its direction cosine with respect to the $z$-direction is uniformly distributed in the interval $\left(\cos \left(\theta_{\max }\right), 1\right)$. That is, simulated realization of $\hat{v}$ fall within a cone. The location of the UCN produced by a neutron is $\left(x_{o}, y_{o}, z_{o}\right)+L_{\text {sim }} \hat{v}$ where $\left(x_{o}, y_{o}, z_{o}\right)$ is the simulated location of the neutron at $z_{o}=-60 \mathrm{~cm}$, and $L_{\text {sim }}$ is the simulated distance traveled by the neutron before a $\mathrm{UCN}$ is produced. Given the initial location of the UCN, we simulate its initial velocity as described earlier. Given the initial velocity and position of an UCN, we determine its trajectory with the symplectic integration method described in Section 3.1.

For the case of no beam divergence, the production rate of simulated above threshold UCNs, $r_{+}$(Eq. 16), produced in the trap at random times in the interval $\left(0, t_{L}\right)$ is 4.2 times larger than the production rate, $r_{-}$(Eq. 15), of simulated below threshold UCNs for the nominal trapping volume defined by $-37.5 \mathrm{~cm} \leq z \leq 37.5 \mathrm{~cm}$ (Figure 4 ). The energy range of simulated above threshold UCNs is $139 \mathrm{neV}$ to $246 \mathrm{neV}$. In general, the UCN wall collision rate increases with energy (Figure 5). Based on Monte Carlo estimates of $S_{M}(t)$ at discrete times $(0,1,2, \cdots, 5500) \mathrm{s}$, we determine $\Delta(t)$ (Eq. 20) on a discrete time grid (Figure 6). Recall, the theoretical value of $\Delta\left(t_{L}\right)$ equals $\frac{\left\langle N_{+}\left(t_{L}\right)\right\rangle}{\left\langle N_{-}\left(t_{L}\right)\right\rangle}$. Even though the relative production of above and below threshold $\mathrm{UCNs}$ is $4.2, \frac{\left\langle N_{+}\left(t_{L}\right)\right\rangle}{\left\langle N_{-}\left(t_{L}\right)\right\rangle}$ is approximately 0.41 . That is, a large fraction of the above threshold UCNs is lost during the filling stage. Since UCNs with energy greater than $246 \mathrm{neV}$ would be lost to the walls in approximately a few seconds or less, such high energy UCNs would have a negligible effect on $\Delta(t)$ for $t-t_{L}>10 \mathrm{~s}$. Thus, extending the maximum energy of simulated above threshold UCN would have a negligible effect on our estimate of $\lambda_{*}$.

We average 40 independent estimates of $S_{M}(t)$ from independent Monte Carlo experiments to get an overall estimate of $S_{M}(t)$ and $\Delta(t)$. Given a wall loss probability model that accounts for incoherent scattering, we determine the mean lifetime of the neutron in the trap to be $700 \mathrm{~s}$ with a standard uncertainty of $57 \mathrm{~s}$ (Table 1, Figure 7). In this standard approach, the model is assumed to be valid and deviations between observations and predicted values based on perfect knowledge of the model parameters are due to counting statistics. To quantify the component of uncertainty due to imperfect 
knowledge of $S_{M}(t)$ due to sampling variability, we simulated bootstrap [27] replications of our estimate of $S_{M}(t)$ by resampling with replacement the 40 independent estimates of $S_{M}(t)$ that we averaged in the first study. For each bootstrap replication of the average $S_{M}(t)$, we refit our model to the same observed data. The bootstrap estimate of uncertainty due to sampling variability of $S_{M}(t)$ is $2.4 \mathrm{~s}$. We note that this uncertainty is very slight compared to the uncertainty due to Poisson counting statistics (approximately $60 \mathrm{~s}$ ) in the observed data. That is, we are quantifying the component of uncertainty in $\lambda_{*}$ due solely to imperfect knowledge of $S_{M}(t)$ here.

\subsection{Systematic effects}

Below, we estimate various systematic effects on the estimate of the neutron lifetime parameter $\tau_{*}$ (Eq. 14). First, we neglect wall loss effects, i.e., we set $\Delta(t)=0$. For this case, we estimate $\tau_{*}$ to be $655 \mathrm{~s}$ with an estimated uncertainty of $51 \mathrm{~s}$ (Table 1). Since we estimate the $\tau_{*}$ to be $700 \mathrm{~s}$ when we account for the wall loss mechanism, we estimate the effect of marginally trapped neutrons on $\tau_{*}$ to be $45 \mathrm{~s}$ for this running configuration. When we estimate $S_{M}(t)$ based on a wall loss model that neglects incoherent scattering effect for the cylindrical boundary, we estimate the neutron lifetime to be 708 $\mathrm{s}$ with an associated 1-sigma uncertainty of $59 \mathrm{~s}$. Based on this, we estimate the component of uncertainty contributed by imperfect knowledge of the wall loss probability model to be $8 \mathrm{~s}$. We emphasize that the above systematic effect can be reduced by purging marginally trapped neutrons from the trap by field ramping techniques as discussed in Section 4 .

For the primary wall loss model that accounts for incoherent scattering, we estimate $\lambda_{*}$ for different definitions of the trapping volume. In particular, we vary the lower axial boundary of the trapping volume, $z_{\text {min }}$, from -37.5 $\mathrm{cm}$ to $-35 \mathrm{~cm}$ and $-40 \mathrm{~cm}$ (Table 2). We fit a linear model to predict the expected value of $\hat{\lambda}$ as a function of $z_{\text {min }}$. The estimated slope of this linear model and its associated uncertainty are $-0.55 \mathrm{~s} / \mathrm{cm}$ and $0.40 \mathrm{~s} / \mathrm{cm}$.

In a similar study, we vary the time-step parameter in the symplectic integration algorithm (Table 2). The estimated slope of a linear model to predict the lifetime as a function of the time step is $2.9 \times 10^{-4}$ where the uncertainty is $3.79 \times 10^{-4}$. Based on this result, the expected difference in a lifetime estimate based on a simulation where the time step parameter $\mathrm{d} t=$ $5.0 \times 10^{-5}$ and where $\mathrm{d} t=\epsilon$ where $\epsilon$ is arbitrarily close to $0 \mathrm{~s}$, is $1.4(1.9) \mathrm{s}$.

Variation in the beam divergence parameter $\theta_{\max }$ produces a statistically significant variation in the lifetime estimate (Figure 8) because the distribu- 
tion of the initial potential energy of an UCN created in the trap depends on $\theta_{\max }$. Thus, even though the distribution of the initial kinetic energy of an UCN produced in the trap does not depend on $\theta_{\max }$, the distribution of the initial total energy of the UCN depends on $\theta_{\text {max }}$. We estimate $\theta_{\max }$ to be 3.0 degrees by matching the expected value of $\theta$ in the simulation to that predicted based on analysis of the dispersion of scattering plane orientations in mosaic crystals relative to their mean value [28]. In [28], the angle between the random orientation of a particular scattering plane and the mean orientation is a truncated Gaussian. Since the slope of the fitted line in Figure 8 is $-0.97(0.16) \mathrm{s} \mathrm{deg}^{-1}$, we estimate the systematic error due to ignoring beam divergence effects to be approximately $2.9 \mathrm{~s}$. As a caveat, to estimate an uncertainty, we assume a one-to-one relationship between the standard deviation of the random mosaic crystal orientations and the standard deviation of direction cosines in our simulation study. Further, in our beam divergence study, the probability distribution function for the direction cosine of a UCN at $z=-60 \mathrm{~cm}$ is a uniform distribution. In contrast, for the mosaic crystal model, the angle between the mean orientation and any random orientation is a truncated Gaussian distribution.

Our estimate of $\lambda_{*}$ systematically depends on the assumed model for how surviving neutrons are reflected back into the trapping volume (Table 3). Because of surface roughness effects, the two diffuse models are much more plausible than than the specular model. Hence, the difference between the estimates for the diffuse models ( $1.3 \mathrm{~s}$ with an uncertainty of $1.5 \mathrm{~s}$ ) is relevant for estimation of a systematic uncertainty due to imperfect knowledge of the model for neutron reflection.

In all earlier studies in this work, we account for gravity and spatial variation in the cold beam fluence. Failure to account for gravity shifts the estimate of $\tau_{*}$ downward by $5.1 \mathrm{~s}$ (standard uncertainty is $1.6 \mathrm{~s}$ ) (Table 4). We expect this shift for two reasons. First, gravity changes the distribution of the initial potential energy of UCNs and hence the distribution of the initial energy of UCNs. Second, for the cases studied here, gravity reduces the minimum potential energy on the nominal trapping volume boundary from $139 \mathrm{neV}$ to $135.5 \mathrm{neV}$. Recall, this minimum potential energy is the threshold for defining a marginally trapped UCN.

Failure to account for spatial variation in assumed neutron beam fluence shifts the estimate of $\tau_{*}$ upward by $7.5 \mathrm{~s}$ (standard uncertainty is $1.6 \mathrm{~s}$ ) (Table 4). As a summary, we list estimates of systematic uncertainties associated with the effects studied here in Table 5. We expect that these systematic 
Table 1. Model parameter estimates for NIST experimental data for a static potential. Survival probability function associated with wall loss determined for two scenarios; incoherent scattering of neutrons in materials is either neglected or accounted for. Beam divergence neglected. The time step parameter for all cases is $\mathrm{d} t=5.0 \mathrm{e}-05 \mathrm{~s}$.

\begin{tabular}{ccc|ccccc}
\hline \hline & & & & & & & \\
case & $\begin{array}{c}\text { account for } \\
\text { incoherent } \\
\text { scattering }\end{array}$ & $\begin{array}{c}\text { account for } \\
\text { marginally } \\
\text { trapped neutrons }\end{array}$ & & & & & \\
& & & & & & \\
a & yes & yes & $57943(3883)$ & $700(57)$ & $1.8(1.8)$ & 1.080 & 0.2234 \\
b & yes & no & $76114(4531)$ & $655(51)$ & $2.3(1.7)$ & 1.085 & 0.2118 \\
c & no & yes & $41558(2856)$ & $708(59)$ & $1.8(1.8)$ & 1.082 & 0.2187 \\
\hline
\end{tabular}

effects can be reduced by purging above threshold UCN from the trap by field ramping strategies as discussed in see Section 4.

For the NIST experiment, we expect the walls of the trap to vibrate and perturb the energy of UCNs that survive wall collisions and are reflected back into the trapping volume. For more discussion of this effect for a simplified 1-D vibrational model for a magneto-gravitational trap, see Salvat and Walstrom [29]. Since the wall loss probability of an UCN depends on its $E_{\perp}$ value, energy perturbations would affect the survival probability of above threshold UCNs for a static trap experiment. Energy perturbations due to mechanical vibrations would also affect how well above threshold UCNs are purged (and how well below threshold UCNs are retained) when magnetic fields are ramped. Development of a realistic three-dimensional model for the effect of mechanical vibrations for the NIST experiment is an important and very challenging research topic beyond the scope of this study.

\section{Purging above threshold UCNs}

In magnetic trapping experiments, one can purge above threshold UCNs from the trap by ramping the trapping potential down and then ramping it back up to its original value $[4,30]$. In a background-free simulation experiment, we reduced the quadrupole field from its maximum value to an adjustable fraction of its initial value (Figure 9). We denote this fraction as 
Table 2. For a static potential experiment, dependence of estimated $\tau_{*}$ on lower axial boundary of nominal trapping volume $\left(z_{\text {min }}\right)$ and time-step $\mathrm{d} t$ in symplectic integration algorithm. We account for wall losses when estimating $\tau_{*}$. Beam divergence effects neglected. Reported uncertainties account for uncertainty of $\Delta(t)$ (Eq. 20) due to Monte Carlo sampling variability.

\begin{tabular}{ccc}
\hline \hline $\mathrm{d} t(\mathrm{~s})$ & $z_{\min }(\mathrm{cm})$ & $\tau_{*}(s)$ \\
& & \\
& & \\
$1.0 \mathrm{e}-04$ & -40 & $703.3(1.5)$ \\
$1.0 \mathrm{e}-04$ & -35 & $700.5(1.5)$ \\
$1.0 \mathrm{e}-04$ & -37.5 & $701.9(1.1)$ \\
$5.0 \mathrm{e}-05$ & -37.5 & $699.9(2.4)$ \\
$2.5 \mathrm{e}-05$ & -37.5 & $699.9(2.8)$ \\
\hline
\end{tabular}

Table 3. For a static potential experiment, dependence of estimated $\tau_{*}$ on neutron scattering modeling. Beam divergence neglected. Reported uncertainties account for uncertainty of $\Delta(t)$ (Eq. 20) due to Monte Carlo sampling variability.

\begin{tabular}{cccc}
\hline \hline & & & \\
reflection model & $\mathrm{d} t(\mathrm{~s})$ & $z_{\min }(\mathrm{cm})$ & $\tau_{*}(s)$ \\
& & & \\
diffuse Lambertian & $1.0 \mathrm{e}-04$ & -37.5 & $701.9(1.1)$ \\
diffuse uniform & $1.0 \mathrm{e}-04$ & -37.5 & $703.2(1.0)$ \\
specular & $1.0 \mathrm{e}-04$ & -37.5 & $713.5(1.5)$
\end{tabular}


Table 4. For a static potential experiment, dependence of estimated $\tau_{*}$. on gravity and spatial variation of neutron beam (Figure 2). For all cases, the wall loss probability model accounts for incoherent scattering. We assume a diffuse Lambertian model for neutron reflections. No beam divergence. The time step parameter is $\mathrm{d} t=1.0 \mathrm{e}-04$. The uncertainties account for imprecise knowledge of the $\Delta(t)$ (Eq. 20) due to Monte Carlo sampling variability.

\begin{tabular}{ccc}
\hline \hline & & \\
gravity accounted for & beam profile accounted for & $\tau_{*}(s)$ \\
yes & yes & $701.9(1.1)$ \\
no & yes & $694.4(1.2)$ \\
yes & no & $707.0(1.2)$
\end{tabular}

Table 5. For a static potential experiment, systematic effects and associated uncertainties for $\tau_{*}$ measurement due to wall losses of marginally trapped UCNs. Effects that are not statistically significant are indicated with asterisks.

\begin{tabular}{ccc}
\hline \hline effect & correction & uncertainty \\
& & \\
wall loss model & none & $8 \mathrm{~s}$ \\
neutron reflections model & none & $1.3 \mathrm{~s}$ \\
beam divergence & -2.9 & $2.9 \mathrm{~s}$ \\
beam profile model & none & $\mathrm{NA}$ \\
upscattering & none & $<<1 \mathrm{~s}$ \\
${ }^{3}$ He absorption & $\mathrm{NA}$ & $\mathrm{NA}$ \\
mechanical vibrations & none & $\mathrm{NA}$ \\
time step & none & $1.9 \mathrm{~s}^{*}$ \\
choice of $z_{\min }$ & none & $1 \mathrm{~s}^{*}$ \\
\hline Total & $-2.9 \mathrm{~s}$ & $8.9 \mathrm{~s}$ \\
\hline \hline
\end{tabular}


a ramping fraction. For any given ramping schedule, we determined the conditional survival probability for all UCNs (rather than just above threshold UCNs) that survive ramping by a direct simulation method for a fixed value of $\tau_{*}=686 \mathrm{~s}$. Based on this conditional survival probability, we simulate high-count $\beta$-decay data under the assumption that the observation rate in narrow $1 \mathrm{~s}$ width bins is well approximated as $\langle N(t)\rangle / \tau_{n}$. We fit the Eq. 26 prediction model to simulated data with $\Delta(t)=0$. That is, we ignore wall losses. For ramping fractions less than approximately 0.3 , the systematic error of the estimated lifetime is very small (Figure 10).

The benefits of purging above threshold UCNs by field ramping come with a cost. That is, we also purge a non-negligible fraction of initially below threshold UCNs. For instance, for the case where the ramping fraction is 0.3 , the fraction of surviving above threshold UCNs immediately after ramping ends is 0.00072 . However, the fraction of originally below threshold UCNs is reduced to $0.30(0.02)$.

There are other field ramping strategies to purge above threshold UCNs than the one considered here. For instance, one could fill the trap with the trapping potential reduced to its minimal value and then ramp it after the filling stage ends. In a simulation experiment, we compared this alternative ramping strategy to ours. We simulated UCNs with the same initial locations and initial velocities and tracked them for both strategies. In order to purge all but 0.0072 of the above threshold UCNs, the fraction of below threshold $\mathrm{UCNs}$ retained by the alternative ramping scheme is $0.06(0.01)$. Thus, for this case, the relative number of below threshold UCNs after ramping is less for the alternative ramping strategy compared to the ramping strategy implemented in our simulation study. To help explain the result, we note that in the alternative ramping schedule, the quadrupole field is maintained at a much lower initial value than in the original ramping schedule. Hence, during the filling stage, the energy threshold that defines a marginally trapped UCN is lower for the alternative ramping scheme compared to the original scheme.

\section{Summary}

We developed a competing risks survival analysis formalism to directly estimate the mean lifetime of a trapped particle in a way that accounts for the joint effects of loss mechanisms with exponential survival probability functions, and a loss mechanism with an non-exponential survival probability function. Our method accounts for effects due to random creation (or 
injection) times of particles in the trap. With this formalism, we analyzed experimental data acquired in a magnetic trapping experiment at NIST. We determined the survival probability function associated with the wall loss mechanism by a Monte Carlo method based on physical models for the loss probability of above threshold UCNs that interact with materials in cylindrical wall and endcaps of the trap. Based on a fit of this model to a subset of the NIST experimental data, we determined the mean lifetime of neutrons in the trap, $\tau_{*}$ (see Eq. 14), to be $700 \mathrm{~s}$ with an uncertainty of approximately $60 \mathrm{~s}$ - considerably less than the current best estimate of $(880.1 \pm$ 1.1) s. We attribute this discrepancy to an additional energy-independent loss mechanism with a constant hazard function (see Eq. 14). That is $\tau_{*}$ depends on all loss mechanisms with constant hazard functions (or equivalently exponential survival probability functions) rather than just the $\beta$-decay loss mechanism. Accelerator based mass spectroscopy measurements carried out to date, strongly suggest that this addition loss mechanism is associated with ${ }^{3} \mathrm{He}$ impurities. (Because the kinetic energy of the neutrons in the centerof-mass frame is far below the energy of any relevant nuclear resonance, the capture rate of trapped UCNs on ${ }^{3} \mathrm{He}$ nuclei is energy independent and the hazard function associated with this loss mechanism is constant.) This work is the subject of an upcoming paper. In our model, the largest source of systematic uncertainty is associated with imperfect knowledge of the wall loss probability model $(8 \mathrm{~s}$ ) (Table 5 ). If we account for the effect of marginally trapped neutrons for the static case considered here, the estimated lifetime of the neutron is shifted upward by $45 \mathrm{~s}$ relative to the estimate obtained by an analysis that ignores the effect of marginally trapped UCNs (Table 1).

In a Monte Carlo experiment, we demonstrated that when the trapping potential is ramped down and then back again, systematic error due to wall losses of marginally trapped UCNs can be suppressed to a very low level. For a particular case, Monte Carlo simulation studies showed that this ramping strategy is more efficient than one where neutrons are trapped when the field is fully ramped and then increased to its maximum value. From a practical perspective, our stochastic model and associated Monte Carlo methods should be helpful to guide design of field ramping strategies to purge above threshold UCNs in magnetic trapping and related trapping experiments.

We expect that the systematic effects we considered for the NIST experiment, and our theoretical survival analysis approach, to be relevant to other neutron lifetime experiments. To apply our methods to other experiments, one would require a model ofr the survival probability function associated 
with an "non-exponential" loss mechanism and perhaps a model for the times at which particles appear in the trap. For analysis of the NIST experiment, to speed up Monte Carlo simulations, we split the population of neutrons into two populations where one corresponded to neutrons that are affected loss mechanisms with associated exponential survival probability functions, and the other corresponded to neutrons affected by a wall loss mechanism with a non-exponential survival probability function. This splitting scheme may find application in other experiments. However, even if such a split is not feasible, our general method should still apply. 
Acknowledgments. We thank Grace Yang for useful comments. We acknowledge the support of the NIST, US Department of Commerce, in providing support, including the neutron facilities used in this work. This work is also supported in part by the US National Science Foundation under Grant No. PHY-0855593 and the US Department of Energy under Grant No. DEFG02-97ER41042. Contributions by staff of NIST, an agency of the US government, are not subject to copyright in the US.

[1] J. Beringer et al. (Particle Data Group), PR D86, 010001 (2012) (URL: http://pdg.lbl.gov)

[2] A. T. Yue, M. S. Dewey, D. M. Gilliam, G. L. Greene, A. B. Laptev, J. S. Nico, W. M. Snow, and F. E. Wietfeldt Phys. Rev. Lett. 111, 222501 (2013)

[3] F.E. Wietfeldt and G.L. Greene, Rev. of Mod. Phys. 83831173 (2011)

[4] S. Paul, Nucl. Instr. and Methods A611 (2009) 157.

[5] H. P. Mumm, M. G. Huber. A. T. Yue, A. K. Thompson, M. S. Dewey, C. R. Huffer, P. R. Huffman, K. W. Schelhammer, C. OShaughnessy and K. J. Coakley, Measuring the Neutron Lifetime with Magnetically Trapped Ultracold Neutrons, 2012 Next Generation Experiments to Measure the Neutron Lifetime Workshop, World Scientific, pp. 121-134, 2014

[6] P. R. Huffman, C. R. Brome, J. S. Butterworth, K. J. Coakley, M. S. Dewey, S. N. Dzhosyuk, R. Golub, G. L. Greene, K. Habicht, S. K. Lamoreaux, C. E. H. Mattoni, D. N. McKinsey, F. E. Wietfeldt, J. M. Doyle Nature 403, 62-64 (6 January 2000)

[7] R. Picker, I. Altarev, J. Brcker, E. Gutsmiedl, J. Hartmann, A. Mller, S. Paul, W. Schott, U. Trinks, and O. Zimmer, J. Res. Natl. Inst. Stand. Technol. 110, 357-360 (2005)

[8] K. Leung and O Zimmer, Nucl. Instr. Methods A611 (2009) 181.

[9] P.L. Walstrom,J.D. Bowman, S.I. Penttila, C. Morris, A. Saunders, Nucl. Instr. Methods A599 (2009) 82.

[10] M. Gail, Biometrics, 209-222. (1975). 
[11] R. L. Prentice, J. D. Kalbfleisch, A. V. Peterson, Jr., N. Flournoy, V. T. Farewell and N. E. Breslow, Biometrics, Vol. 34, No. 4 (Dec., 1978), pp. $541-554$

[12] J. D. Kalbfleisch and R. L. Prentice The Statistical Analysis of Failure Time Data, Second Edition, John Wiley \& Sons, Inc. 2002

[13] H. Putter, M. Fiocco and R. B. Geskus, Statist. Med. 2007; 26:23892430

[14] R. Golub and J.M. Pendlebury, Phys. Lett. 53A, 133 (1975).

[15] R. Golub, D. Richardson, S.K. Lamoreaux, Ultra-Cold Neutrons, Taylor and Francis, 1991

[16] A. Steyerl, S. S. Malik, A. M. Desai, and C. Kaufman, Phys. Rev. C 81, 055505 (2010)

[17] C.M. Oshaughnessy, PhD thesis, North Carolina State University, 2010

[18] K.J. Coakley, Stochastic modeling and simulation of marginally trapped neutrons, 2012 Next Generation Experiments to Measure the Neutron Lifetime Workshop, World Scientific, pp. 65-74, 2014

[19] K. J. Coakley, J. M. Doyle, S. N. Dzhosyuk, L. Yang, P. R. Huffman, J. Res. Natl. Inst. Stand. Technol. 110, 367-376 (2005)

[20] R. I. McLachlan and P. Atela, Nonlinearity 5, 541-562 (1992).

[21] P.J. Channel and C. Scovel, Nonlinearity 3, 231 (1990).

[22] C. de Boor, A practical guide to splines, Springer-Verlag, New York (1978).

[23] E. Korobkina, R. Golub, J. Butterworth, P. Geltenbort, and S. Arzumanov, Temperature dependence of ultracold neutron loss rates, Phys. Rev. B 70 (3) (2004) 035409

[24] C. M. Goral, K. E. Torrance, D. P. Greenberg and B. Battaile, Modeling the Interaction of Light Between Diffuse Surfaces, Comput. Graphics, Volume 18, Number 3, (1984), pp. 213-222. 
[25] J. von Neumann, "Various techniques used in connection with random digits. Monte Carlo methods", J. Res. Natl. Bur. Stand., 12 (1951), pp. 3638.

[26] Robert, C.P. and Casella, G. "Monte Carlo Statistical Methods" (second edition). New York: Springer-Verlag, 2004.

[27] B. Efron are R.J. Tibshirani, An Introduction to the Bootstrap, Monographs on Statistics and Applied Probability, Chapman and Hall/ CRC Press, (1993)

[28] C.E.H. Mattoni, C.P. Adams, K.J. Alvine, J.M. Doyle, S.N. Dzhosyuk, R. Golub , E. Korobkina, , D.N. McKinsey, A.K. Thompson, , L. Yang H. Zabel P.R. Huffman, A long wavelength neutron monochromator for superthermal production of ultracold neutrons Physica B 344 (2004) 343-357

[29] D.J. Savat and P.L. Walstrom, Vibration-induced loss of ultra-cold neutrons in a magneto-gravitational trap, 2012 Next Generation Experiments to Measure the Neutron Lifetime Workshop, World Scientific, pp. 87-96, 2014

[30] L. Yang, PhD thesis, Harvard University, 2006. 


\section{FIGURES}
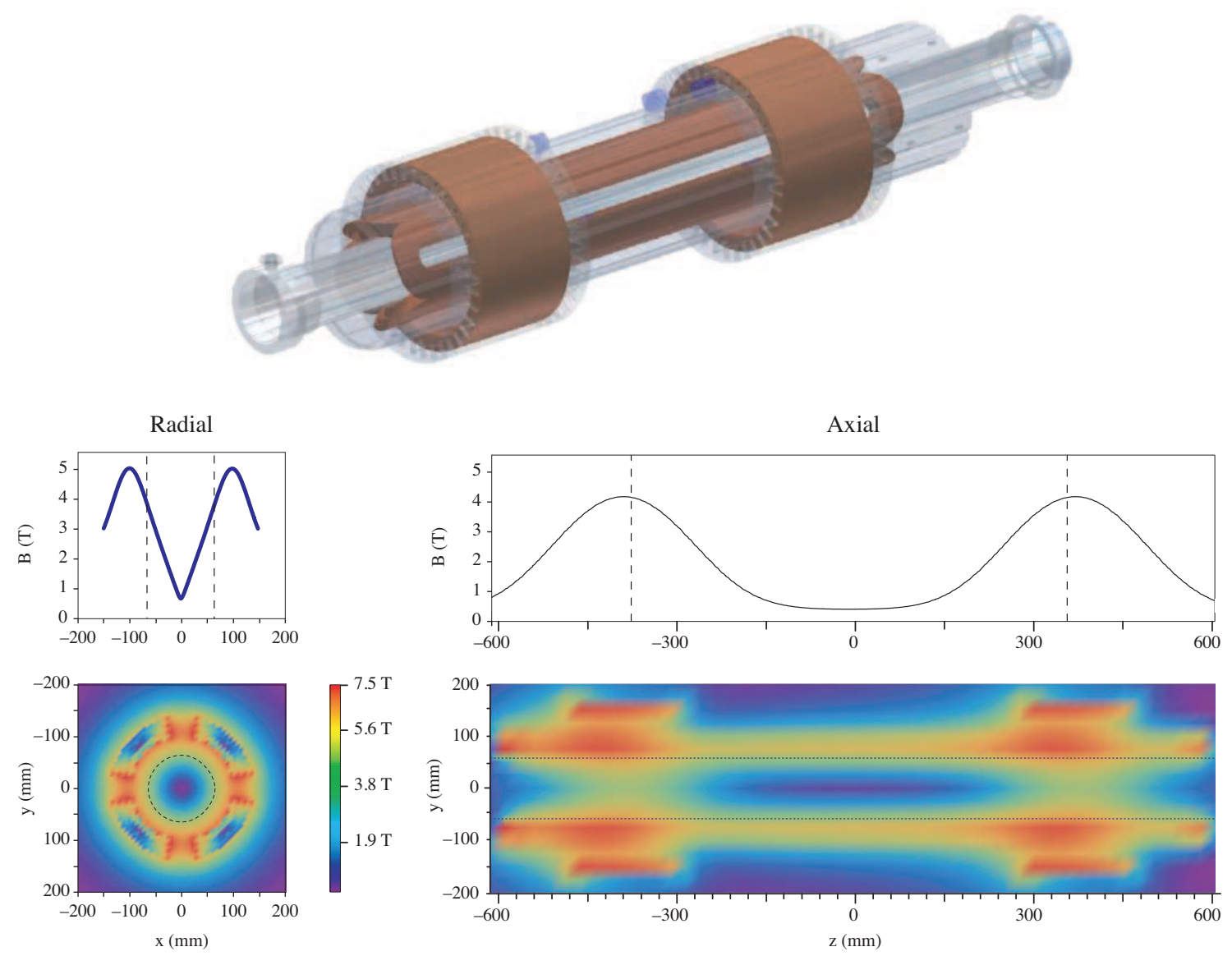

Figure 1: Magnetic field profiles in the 27 IST magnetic trapping experiment. 
(a)

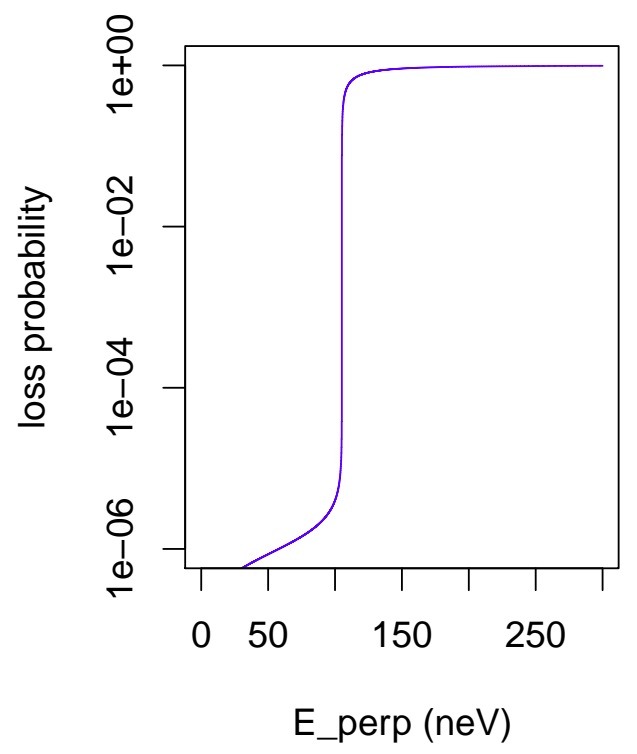

(c)

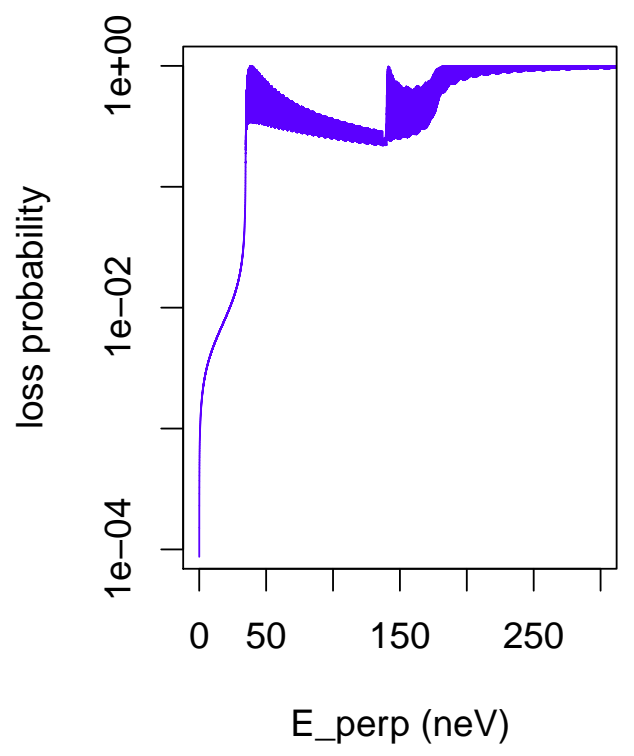

(b)

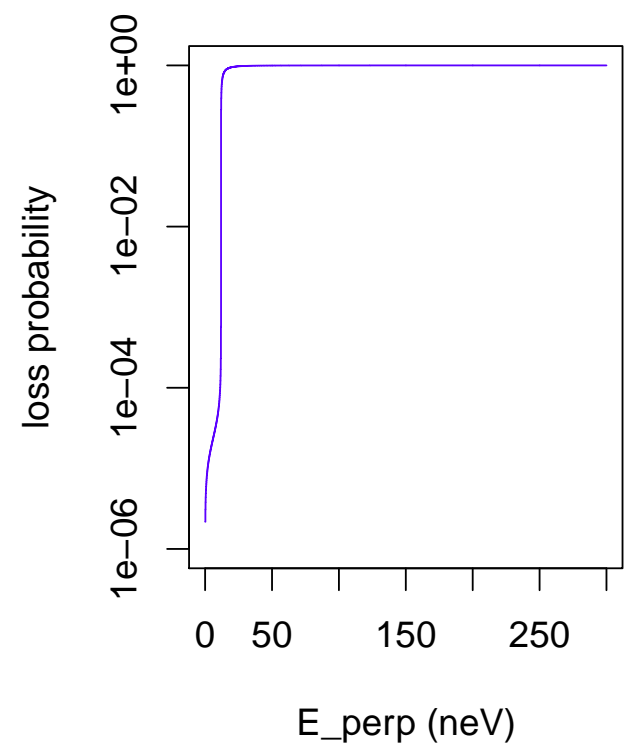

Figure 2: Loss probabilities for (a) the Teflon coated endcap; (b) the acrylic coated endcap and (c) multilayers at cylindrical wall. 


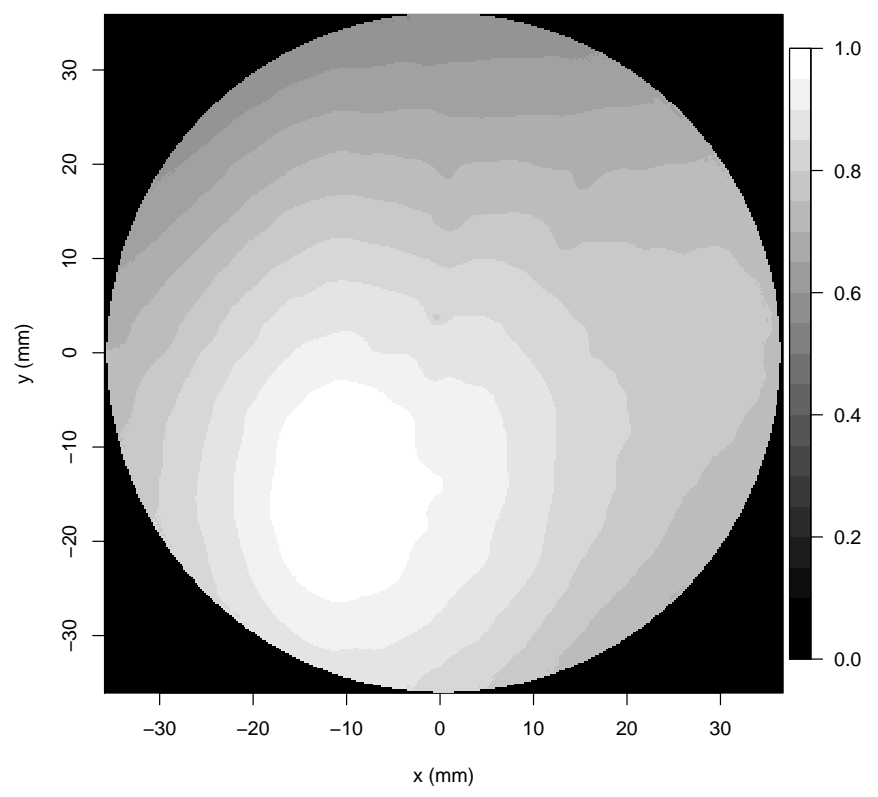

Figure 3: Model for normalized neutron fluence in NIST magnetic trapping experiment at trap entrance. 


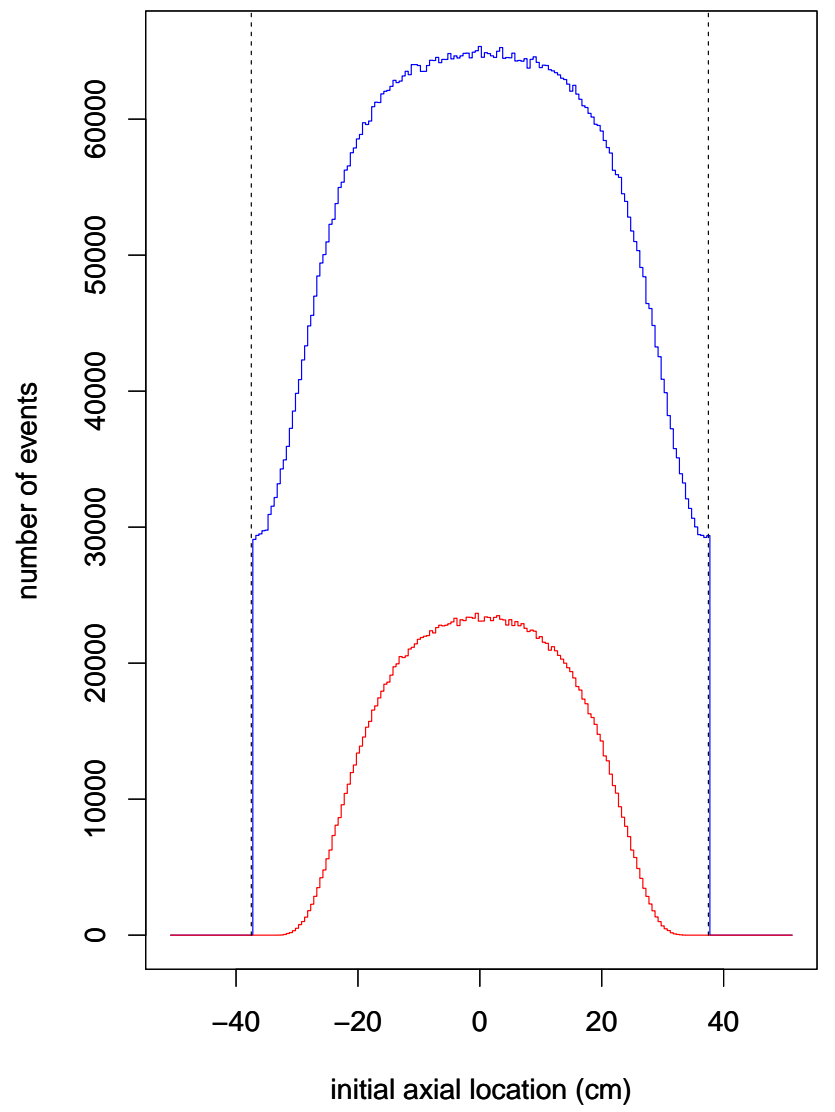

Figure 4: For a static trap where the trapping volume is defined to be $-37.5 \mathrm{~cm} \leq z \leq$ $37.5 \mathrm{~cm}$, the ratio of above threshold to below threshold UCNs is 4.22 for energies between approximately $139 \mathrm{neV}$ to $246 \mathrm{neV}$. 
(a)

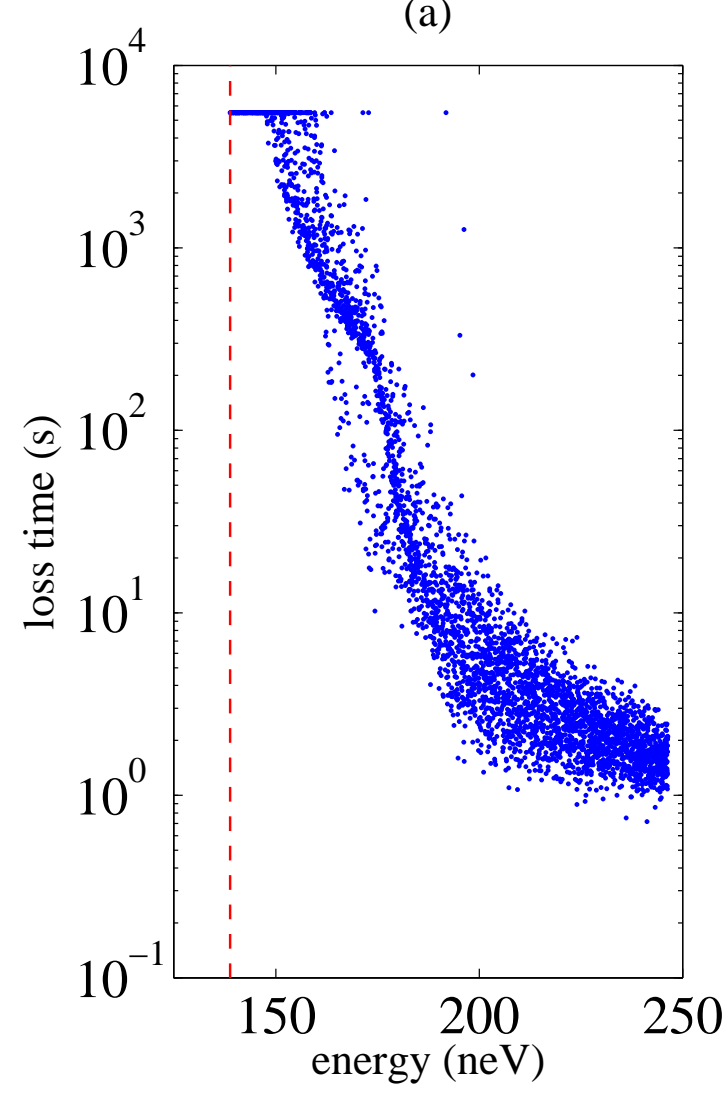

(b)

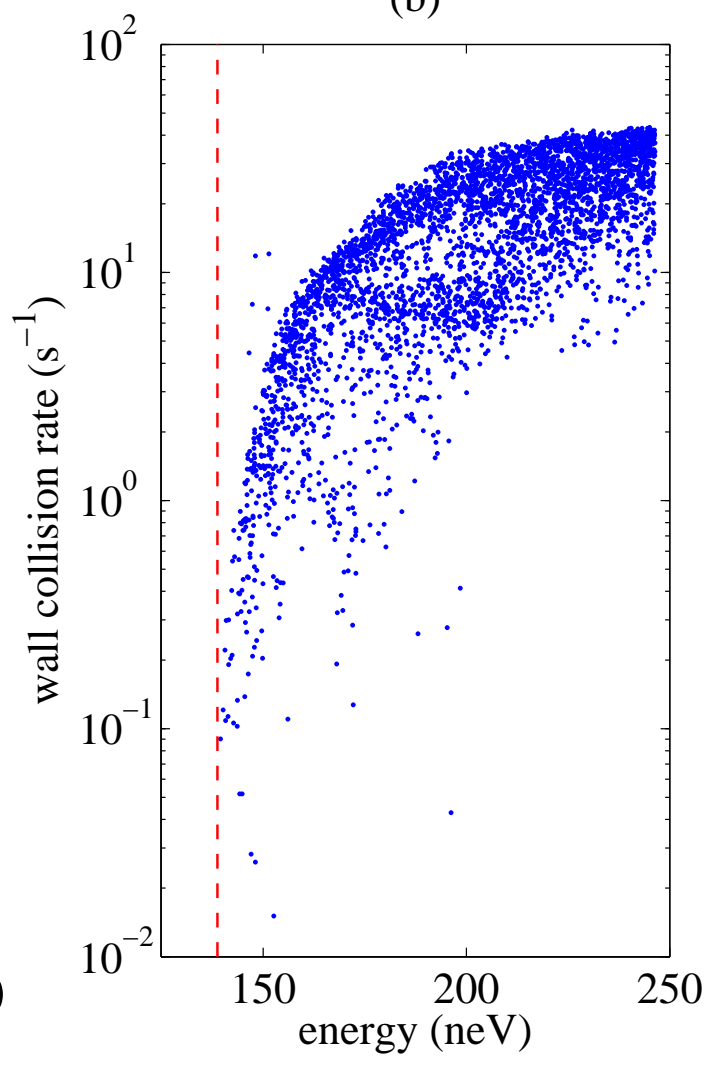

Figure 5: Loss time and wall collision rates 31 or simulated above threshold UCNs. A UCN is lost when its empirical survival probability falls below 1.0e-09. Tracking halted $5500 \mathrm{~s}$ after creation time if a UCN has survival probability above 1.0e-09. 
(a)

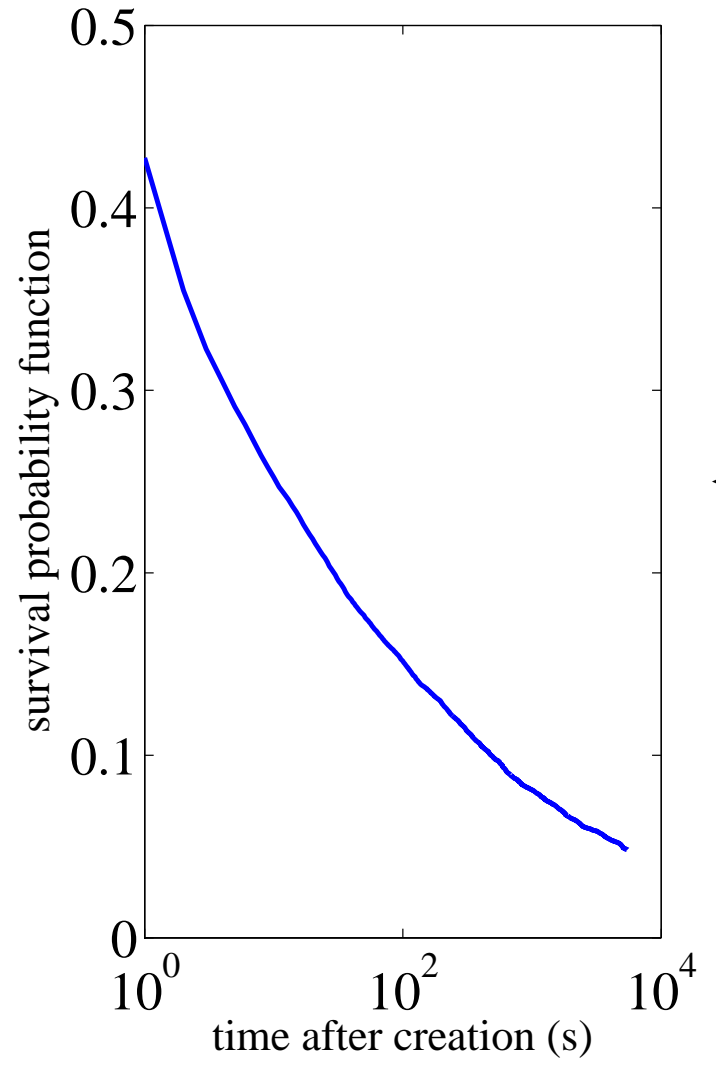

(b)

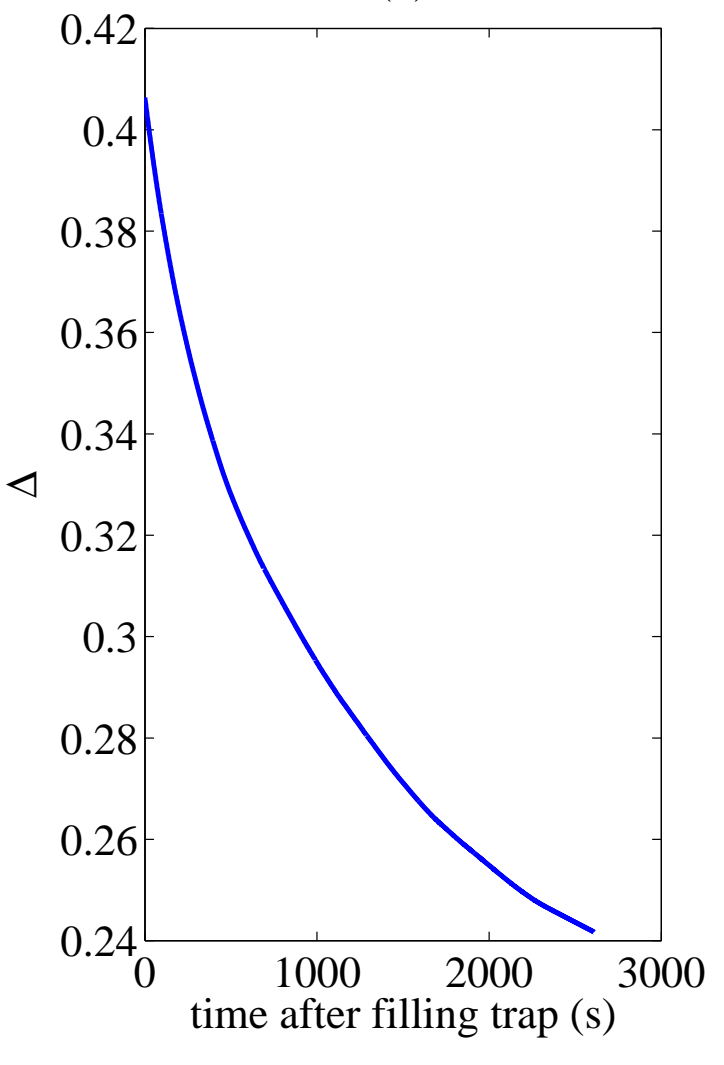

Figure 6: (a). Monte Carlo estimate of survival probability function of above threshold UCNs. (b). Monte Carlo estimate of distortion term $\Delta(t)$ (Eq. 20). 


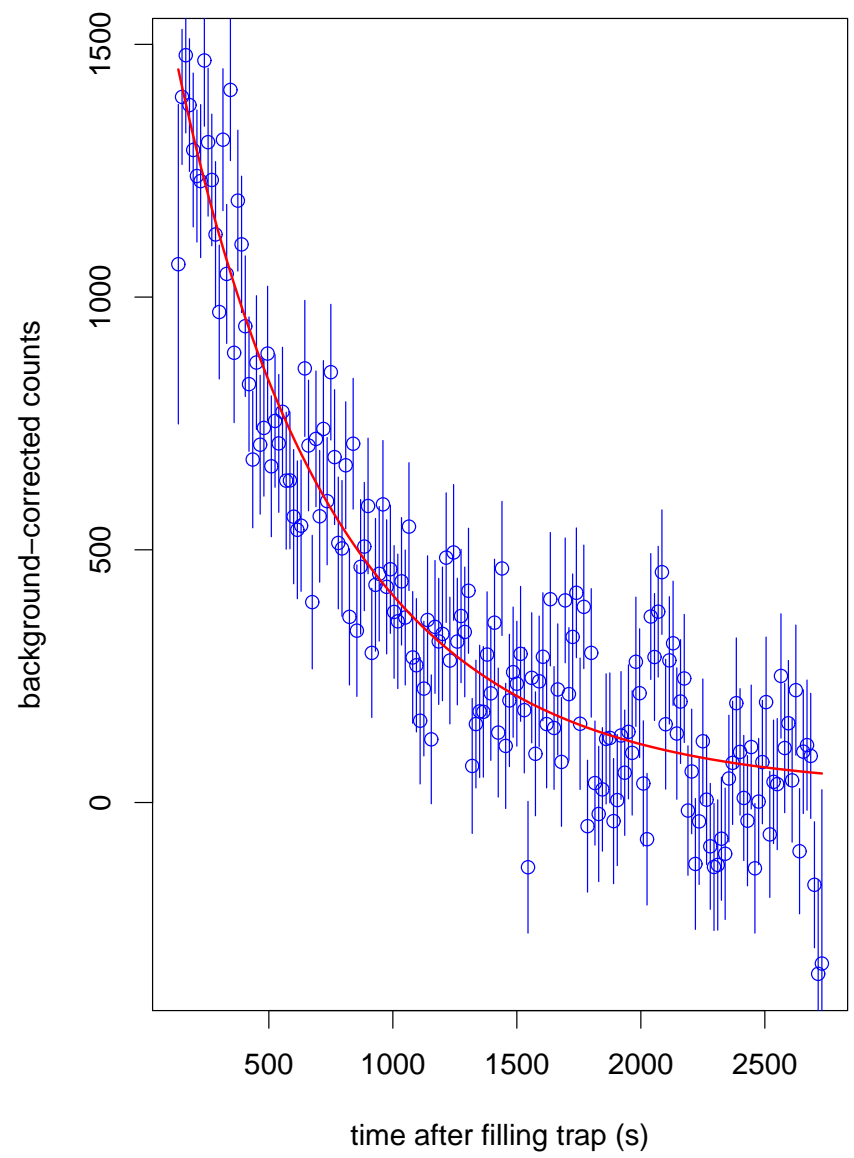

Figure 7: Observed and predicted (line) background-corrected data for a subset of data from the NIST magnetic trapping experiment. For this subset, the trapping potential is static. Prediction model accounts for wall losses. 


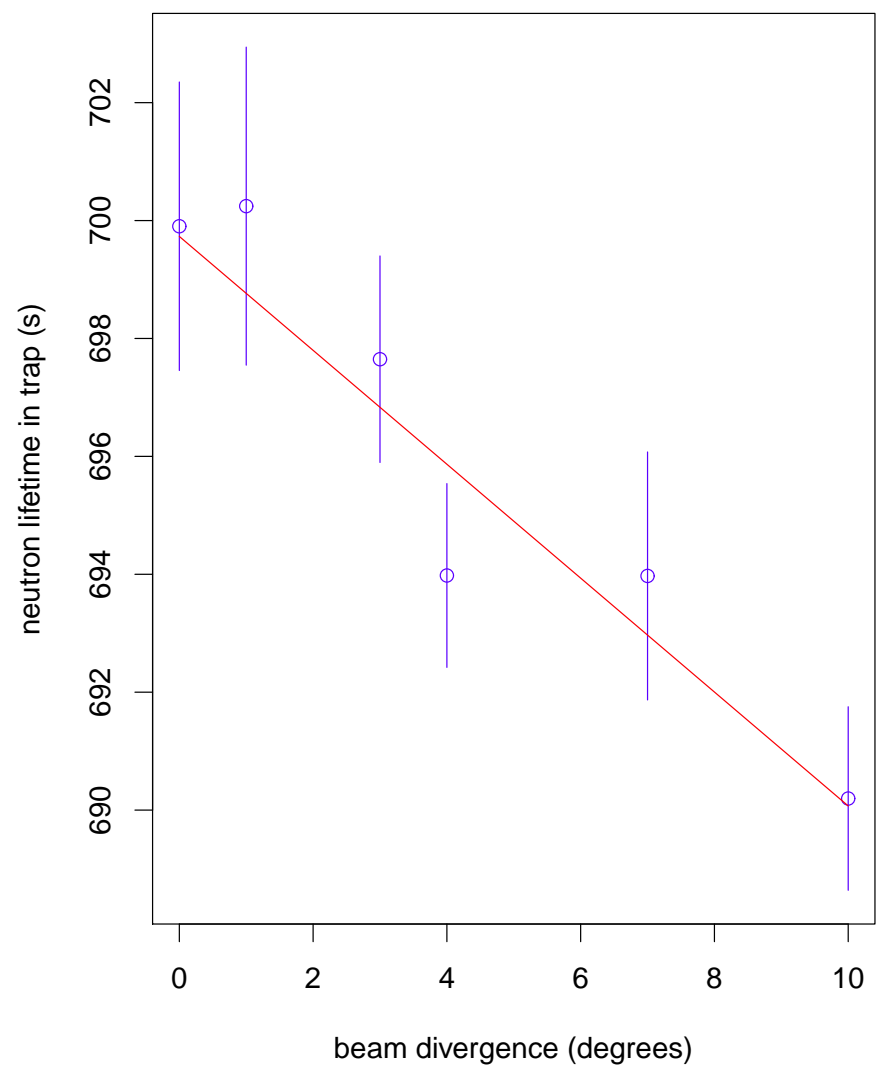

Figure 8: Model Carlo estimates of $\Delta(t)$ depend on assumed beam divergence witin the trap. Fitted intercept and slope are 699.73(1.03) and $-0.97(0.17) \mathrm{s} /$ degree. 


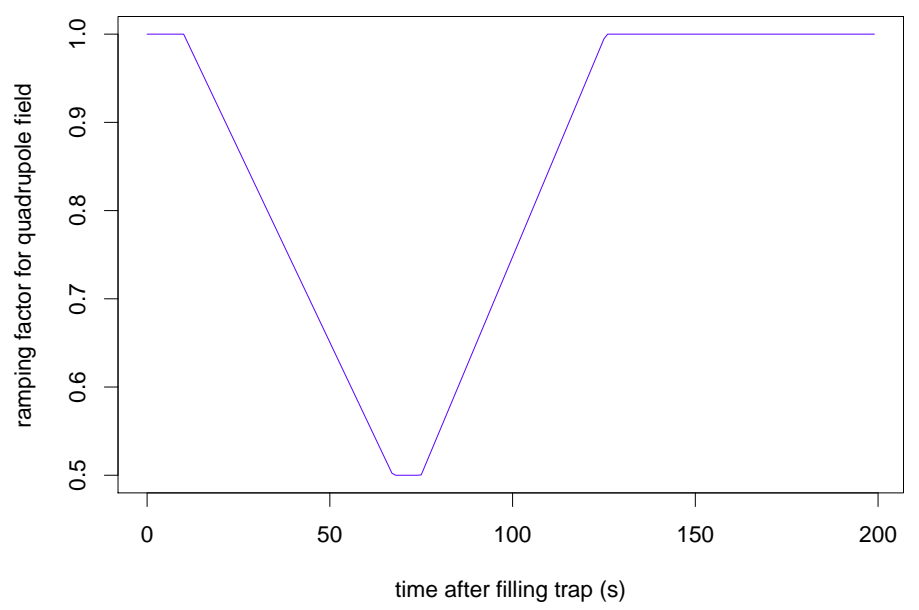

Figure 9: In simulation experiment, the quadrupole field is reduced by a fraction that varies from 1 to an adjustable minimum.

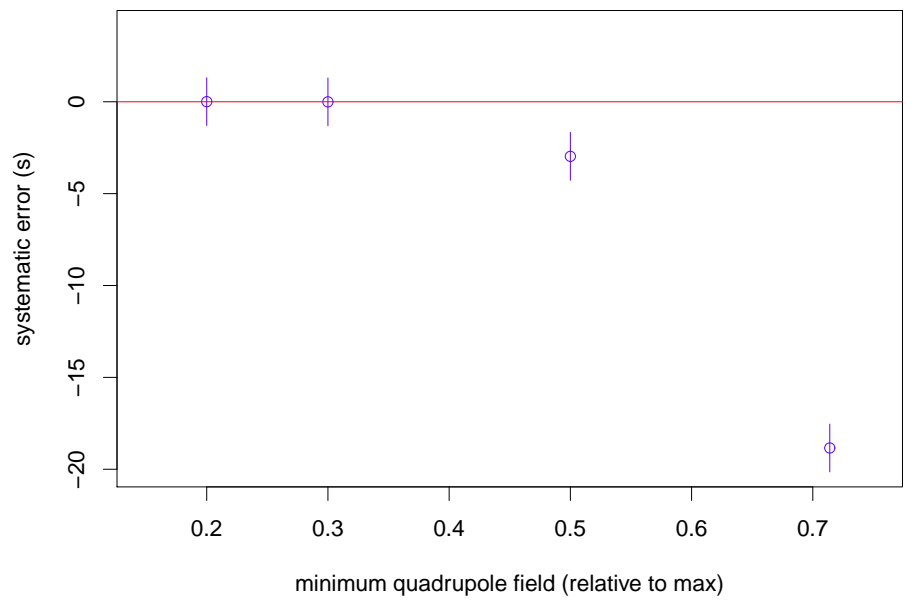

Figure 10: Bias of neutron lifetime when an exponential model is fit to simulated data contaminated by above threshold UCNs. For unramped field, bias is $-31(2) \mathrm{s}$. The true value of $\tau_{*}$ is $686 \mathrm{~s}$. 